\title{
The effect of bone cement distribution on the outcome of percutaneous Vertebroplasty: a case cohort study
}

\author{
Lei Tan ${ }^{1}$, Bingtao Wen ${ }^{1 *}$, Zhaoqing Guo ${ }^{1,2}$ and Zhongqiang Chen ${ }^{1,2}$
}

\begin{abstract}
Background: To analyze the effect of different types of bone cement distribution after percutaneous vertebroplasty (PVP) in patients with osteoporotic vertebral compression fracture (OVCF).

Methods: One hundred thirty seven patients with single level OVCF who underwent PVP were retrospectively analyzed. The patients were divided into two groups according to bone cement distribution. Group A: bone cement contacted both upper and lower endplates; Group B: bone cement missed at least one endplate. Group B was divided into 3 subgroups. Group B1: bone cement only contacted the upper endplates; Group B2: bone cement only contacted the lower endplates; Group B3: bone cement only located in the middle of vertebral body. The visual analogue scale (VAS) score at $24 \mathrm{~h}$ post operation and last follow-up, anterior vertebral height restoration ratio (AVHRR), anterior vertebral height loss ratio (AVHLR), local kyphotic angle change and vertebral body recompression rate were compared.

Results: $24 \mathrm{~h}$ post operation, the pain of all groups were significantly improved. The average follow-up time was $15.3 \pm 6.3$ (6-24) months. At last follow-up, the VAS score of group A was lower than that of group B. There were 14 cases (10.2\%) of adjacent vertebral fracture, 5 cases (8.6\%) in group A and 9 cases (11.4\%) in group B. There were 9 cases (6.6\%) of cement leakage, 4 cases (6.9\%) in group A and 5 cases (6.3\%) in group B. At last follow-up, there were 16 cases (11.7\%) of vertebral body recompression, including 3 cases (5.2\%) in group A and 13 cases (16.5\%) in group B. There was no significant difference in AVHRR between two groups. Local kyphotic angle change was significant larger in group B. At last follow-up, AVHLR in group B was higher than that in group A. Analysis in subgroup $B$ revealed no significant difference in VAS score, local kyphotic angle change, vertebral recompression rate, AVHRR or AVHLR.
\end{abstract}

Conclusions: If the bone cement fully contacted both the upper and lower endplates, it can better restore the strength of the vertebral body and maintain the height of the vertebral body, reduce the risk of the vertebral body recompression and long-term pain.

Keywords: Osteoporotic vertebral compression fracture, OVCF, Percutaneous vertebroplasty, PVP, Bone cement distribution, Vertebral body height, Vertebral body recompression

\footnotetext{
*Correspondence: wenbingtao1101@yeah.net

1 Department of Orthopaedics, Peking University International Hospital, Life

Park Road No.1 Life Science Park of Zhong Guancun, Changping District,

Beijing 102206, China

Full list of author information is available at the end of the article
}

(c) The Author(s). 2020 Open Access This article is licensed under a Creative Commons Attribution 4.0 International License, which permits use, sharing, adaptation, distribution and reproduction in any medium or format, as long as you give appropriate credit to the original author(s) and the source, provide a link to the Creative Commons licence, and indicate if changes were made. The images or other third party material in this article are included in the article's Creative Commons licence, unless indicated otherwise in a credit line to the material. If material is not included in the article's Creative Commons licence and your intended use is not permitted by statutory regulation or exceeds the permitted use, you will need to obtain permission directly from the copyright holder. To view a copy of this licence, visit http://creativecommons.org/licenses/by/4.0/. The Creative Commons Public Domain Dedication waiver (http://creativecommons.org/publicdomain/zero/1.0/) applies to the data made available in this article, unless otherwise stated in a credit line to the data. 


\section{Background}

With the aging of the population, osteoporotic vertebral compression fracture (OVCF) is becoming more common. Several literatures have confirmed that percutaneous vertebroplasty (PVP) is an effective method for the treatment of such fractures, which can effectively relieve pain, maintain the strength of the vertebral body, and avoid long-term complications from bedridden [1, 2]. However, there are complications such as refracture, loss of vertebral body height and increase of local kyphosis angle, which may be affected by the distribution of bone cement in vertebral body during the first operation. The purpose of this study was to analyze the effect of different types of bone cement distribution on pain relief, vertebral height maintenance, and the rate of vertebral recompression.

\section{Methods}

\section{General data}

Patients with OVCF who underwent single level PVP operation in our institute from June 2016 to June 2019 were analyzed retrospectively. Inclusion criteria: (1) patients with lower back pain as the main manifestation, not accompanied by lower extremity radiation pain, numbness, weakness or other nerve compression symptoms; (2) T-score $<-2.5$ in bone mineral density (BMD) examination of lumbar spine, to confirm the osteoporosis; (3) high signal changes in the vertebral body on fat suppression MRI or bone scan examination showed active bone metabolism, to confirm the acute fracture. Exclusion criteria: (1) pathological fracture caused by tumor or infection; (2) patients with severe systemic diseases, unable to tolerate surgery; (3) patients with incomplete data or missing visit. One hundred fifty two patients were initially identified. We enrolled 137 patients and 15 were lost to follow. There were 26 males and 111 females, with an average age of $69 \pm 7.0$ years old. The average follow-up time was $15.3 \pm 6.3$ (6-24) months.

\section{Surgical method}

The patient was placed in the prone position and local anesthesia was performed with $1 \%$ lidocaine. Under the guidance of $\mathrm{C}$-arm fluoroscopy, the puncture needles were placed through bilateral pedicle paths. The end of the puncture needle was located at 1/3 anterior-mid of the vertebral body on lateral film, and between the inner edge of the ipsilateral pedicle and the midline of the vertebral body on the anteroposterior film. Using a hydraulic injection device, the high viscosity cement was injected slowly under fluoroscopy until the cement was close to the posterior wall of the vertebral body which the leakage was possible. According to medical protocol of our institution, all patients who underwent vertebroplasty would take calcium (100 mg per day), calcitriol $(0.5 \mu \mathrm{g}$ per day) and alendronate sodium $(70 \mathrm{mg}$ per week) after surgery. The patients were reminded to take medicine on time on regular visit.

\section{Grouping method}

Radiographs were taken $24 \mathrm{~h}$ post operation and patients were divided into two groups according to the distribution of bone cement. Group A: bone cement contacted both upper and lower endplates. Group B: bone cement missed at least one endplate. Group B was divided into 3 subgroups. Group B1: bone cement only contacted the upper endplates; Group B2: bone cement only contacted the lower endplates; Group B3: bone cement only located in the middle of vertebral body. Figure 1 showed illustrations of cement distribution.

\section{Evaluation method}

Age, gender, body mass index (BMI), BMD, fracture segment, vertebral compression degree (mild $<25 \%$, moderate $26-40 \%$, severe $>40 \%$ ), bone cement volume, adjacent vertebral fracture and bone cement leakage were documented. The visual analog scale (VAS) score pre-operation, $24 \mathrm{~h}$ post operation and at the last followup were analyzed. Radiography measurement indexes include the anterior vertebral height ratio (AVHR), which was defined as the height of the anterior wall of the compressed vertebral body / (the height of the anterior wall of the upper vertebral body + the height of the anterior wall of the lower vertebral body) $\times 2$. The anterior vertebral height recovery ratio (AVHRR) was defined as postoperative AVHR - preoperative AVHR. The anterior vertebral height loss ratio (AVHLR) was defined as postoperative AVHR - last follow-up AVHR. On the last follow-up, a recompression was confirmed when the height of the anterior wall of the vertebral body deceased more than $1 \mathrm{~mm}$ compared with the post operation, or the Cobb angle of the upper and lower endplates increased more than $10^{\circ}$. Local kyphotic angle change was defined as last follow-up Cobb angle of upper and lower endplates - postoperative Cobb angle of upper and lower endplates.

\section{Statistical method}

SPSS 19.0 software (SPSS Inc., USA) was used to analyze the data. The continuous variable was expressed as mean \pm standard deviation, and independent sample $\mathrm{t}$ test or variance analysis (ANOVA) was used. Chi square test was adapted to analyze the categorical variable. Significant differences were defined as $p<0.05$.

\section{Results}

There was no significant difference in age, gender, BMI, $\mathrm{BMD}$, fracture segment, fracture compression degree, 

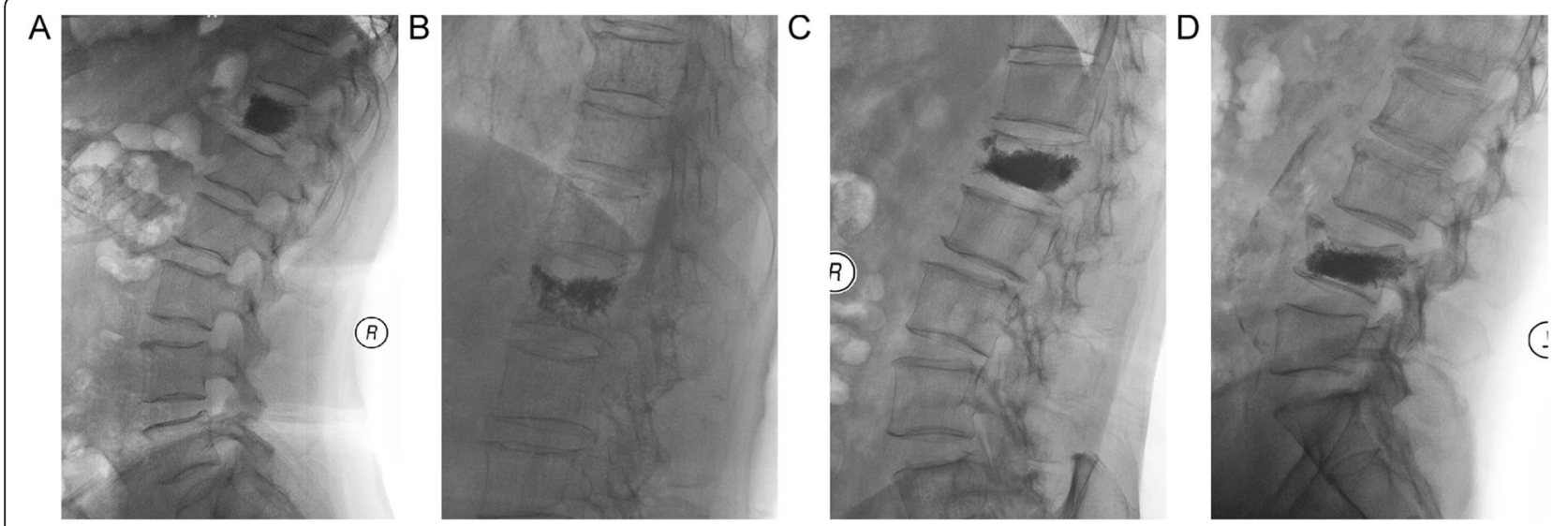

Fig. 1 Different types of bone cement distribution. a. Bone cement is in contact with the upper and lower endplates. b. Bone cement is only in contact with the upper endplate. c. Bone cement is only in contact with the lower endplate. $\mathbf{d}$. Bone cement is not in contact with the endplate

bone cement volume and follow-up time between groups (as shown in Table 1).

The pain was significantly relieved and there was no statistical difference in pre-operation or $24 \mathrm{~h}$ postoperation VAS score between group A and B. At the last follow-up, the VAS score of group A was statistically lower than that of group B. There were 14 cases (10.2\%) of adjacent vertebral fracture, 5 cases $(8.6 \%)$ in group A and 9 cases $(11.4 \%)$ in group B. There were 9 cases (6.6\%) of bone cement leakage, 4 cases $(6.9 \%)$ in group A and 5 cases $(6.3 \%)$ in group B. There was no statistical difference between two groups. At the last follow-up, there were 16 cases $(11.7 \%)$ of vertebral recompression, including 3 cases (5.2\%) in group A and 13 cases (16.5\%) in group B. There was statistical difference between the two groups. Local kyphotic angle change was significant larger in group B. There was no significant difference in AVHRR between the two groups. At the last follow-up, AVHLR in group B was significantly higher than that in group A (Table 2).

Analysis in subgroup B revealed no significant difference in VAS score, adjacent vertebral fracture rate, bone cement leakage rate, local kyphotic angle change, vertebral recompression rate, AVHRR or AVHLR (Table 3). Typical cases were shown in Fig. 2.

\section{Discussion}

OVCF is commonly seen in elderly patients. In a multicenter prospective study involving 2451 elderly women, $32 \%$ of the patients had at least one vertebral

Table 1 Basic information of patients

\begin{tabular}{|c|c|c|c|c|c|}
\hline & Group A $(n=58)$ & Group B1 $(n=30)$ & Group B2 $(n=37)$ & Group B3 $(n=12)$ & $p$ value \\
\hline$\overline{\text { Age }}$ & $68.9 \pm 8.5$ & $69.8 \pm 6.1$ & $69.7 \pm 8.7$ & $74.0 \pm 6.2$ & 0.248 \\
\hline Gender & & & & & 0.188 \\
\hline Male & 15 & 2 & 7 & 2 & \\
\hline Female & 43 & 28 & 30 & 10 & \\
\hline $\mathrm{BMI}\left(\mathrm{kg} / \mathrm{m}^{2}\right)$ & $23.5 \pm 4.3$ & $21.6 \pm 4.7$ & $22.9 \pm 5.2$ & $21.6 \pm 2.9$ & 0.253 \\
\hline Bone mineral density ( $\mathrm{T}$ score) & $-3.2 \pm 0.4$ & $-3.3 \pm 0.3$ & $-3.3 \pm 0.3$ & $-3.4 \pm 0.3$ & 0.325 \\
\hline Fracture segment & & & & & 0.585 \\
\hline Thoracic $(\mathrm{T} 1-10)$ & 9 & 6 & 4 & 3 & \\
\hline Thoracolumbar (T11-L2) & 41 & 22 & 28 & 5 & \\
\hline Lumbar (L3-5) & 8 & 3 & 6 & 3 & \\
\hline Vertebral compression degree & & & & & 0.820 \\
\hline Mild $(<25 \%)$ & 25 & 10 & 14 & 3 & \\
\hline Moderate $(26 \sim 40 \%)$ & 16 & 10 & 12 & 6 & \\
\hline Severe (> 40\%) & 17 & 10 & 11 & 3 & \\
\hline Bone cement volume $(\mathrm{mL})$ & $6.0 \pm 1.3$ & $6.1 \pm 1.0$ & $5.6 \pm 0.9$ & $5.7 \pm 1.1$ & 0.247 \\
\hline Follow up time (months) & $16.2 \pm 6.5$ & $14.7 \pm 6.1$ & $14.0 \pm 5.9$ & $16.0 \pm 7.2$ & 0.374 \\
\hline
\end{tabular}


Table 2 Analysis of outcome between different groups

\begin{tabular}{llll}
\hline & Group A & Group B & $p$ value \\
\hline VAS (pre-op) & $6.1 \pm 1.7$ & $5.7 \pm 1.5$ & 0.157 \\
VAS (24 h post-op) & $2.0 \pm 1.2$ & $1.9 \pm 1.1$ & 0.641 \\
VAS (last follow-up) & $1.4 \pm 1.1$ & $2.0 \pm 1.2$ & $0.002^{*}$ \\
Adjacent vertebral fracture (\%) & $8.6 \%(5 / 58)$ & $11.4 \%(9 / 79)$ & 0.597 \\
Bone cement leakage (\%) & $6.9 \%(4 / 58)$ & $6.3 \%(5 / 79)$ & 0.895 \\
Local kyphotic angle change $\left(^{\circ}\right)$ & $3.4 \pm 3.9$ & $4.9 \pm 4.1$ & $0.029^{*}$ \\
Recompression (\%) & $5.2 \%(3 / 58)$ & $16.5 \%(13 / 79)$ & $0.042^{*}$ \\
AVHRR (\%) & $6.6 \pm 4.0$ & $5.8 \pm 3.9$ & 0.241 \\
AVHLR (\%) & $4.0 \pm 2.6$ & $6.8 \pm 3.8$ & $<0.001^{*}$ \\
\hline *there was statistical difference when $p<0.05$ & &
\end{tabular}

*there was statistical difference when $p<0.05$

compression fracture [3], which may occur with or without slight trauma. The bone cement can restore the strength of the fractured vertebral body and produce thermal necrosis effect on the pain nerves in the vertebral body. It is an effective way to treat OVCF $[4,5]$ and affected by many factors such as the patient's BMD, the volume and distribution of bone cement [6]. Biomechanical tests have shown that restoration of strength and stiffness required vertebral body cement fills of 16.2 and $29.8 \%$, respectively [7]. It is not only the volume of bone cement, but also the distribution of bone cement in the vertebral body has an important influence on the effect of operation and the long-term maintenance of the vertebral body height.

Some literatures classified the bone cement distribution according to its diffusion on anteroposterior X-ray film, and then their influence on the long-term outcome was studied [8]. However, this classification method was mostly applicable for unilateral puncture cases, because bilateral puncture injection of bone cement can often achieve a uniform distribution on both sides of the vertebral body, effectively avoiding the uneven stress caused by asymmetric distribution on the coronal plane. Many previous literatures have also confirmed that bilateral puncture does not significantly increase the risk of complications such as cement leakage and nerve injury as long as the puncture route is strictly followed $[9,10]$. Therefore, studying the distribution of bone cement in the sagittal plane on lateral radiographs may be more meaningful for the analysis of surgical efficacy.

Our study found that if bone cement can fully contact with the upper and lower endplates, it can better maintain the height of the vertebral body and reduce the risk of vertebral recompression. In our study, the surgical vertebral recompression rate in Group A was 5.2\%, which was significantly lower than that in group B (16.5\%). Previous literatures reported a surgical vertebral recompression rate of $3.2 \% \sim 27.6 \%$ with different criteria of recompression and follow-up time [11-13]. This kind of surgical vertebral recompression is multifactorial, often without a clear traumatic event, may be related to the degree of osteoporosis, daily activities and the distribution of bone cement [14]. Insufficient filling of bone cement is an important cause of recompression, especially the uneven distribution in the sagittal plane [15]. If the cement is in full contact with the upper and lower endplates, it will fill the whole vertebral body and play a full role of "bonding" for the cancellous bone and the endplate, which can better restore the strength of the vertebral body [16]. When the cement only touches the upper or lower endplates, vertebral strength only increases about 2 times. If the cement touches the upper and lower endplates at the same time, it will prompt 8 12 times and significantly improve the stress transmission [17]. In Kim's study, 46.7\% (7/15) of the patients have a recompression at an average of 3.4 months post operation if the cement had no contact with the endplate [18]. Our finding is consistent with Liang, after an average follow-up of 29.6 months, $8.16 \%$ or $37.4 \%$ of the patients will have surgical vertebral recompression if the cement is in full contact with the upper and lower endplates or not in their study [19].

Table 3 Analysis of outcome between sub-Group B

\begin{tabular}{lllll}
\hline & Group B1 & Group B2 & Group B3 & $p$ value \\
\hline VAS (pre-op) & $5.8 \pm 1.5$ & $5.8 \pm 1.4$ & $5.3 \pm 1.8$ & $1.6 \pm 1.2$ \\
VAS (24 h post-op) & $2.1 \pm 1.2$ & $1.8 \pm 1.1$ & $1.8 \pm 1.6$ & 0.542 \\
VAS (last follow-up) & $1.7 \pm 1.3$ & $2.4 \pm 1.3$ & $8.3 \%(1 / 12)$ & 0.072 \\
Adjacent vertebral fracture (\%) & $16.7 \%(5 / 30)$ & $8.1 \%(3 / 37)$ & $0 \%(0 / 12)$ & 0.513 \\
Bone cement leakage (\%) & $6.7 \%(2 / 30)$ & $8.2 \%(3 / 37)$ & $5.7 \pm 5.1$ & 0.602 \\
Local kyphotic angle change $\left(^{\circ}\right)$ & $4.7 \pm 3.5$ & $4.8 \pm 4.4$ & $25 \%(3 / 12)$ & 0.777 \\
Recompression (\%) & $13.3 \%(4 / 30)$ & $16.2 \%(6 / 37)$ & $4.7 \pm 4.2$ & 0.653 \\
AVHRR (\%) & $5.4 \pm 3.8$ & $6.6 \pm 3.7$ & $7.1 \pm 2.5$ & 0.248 \\
AVHLR (\%) & $7.6 \pm 4.0$ & $6.0 \pm 3.9$ & & 0.213 \\
\hline
\end{tabular}



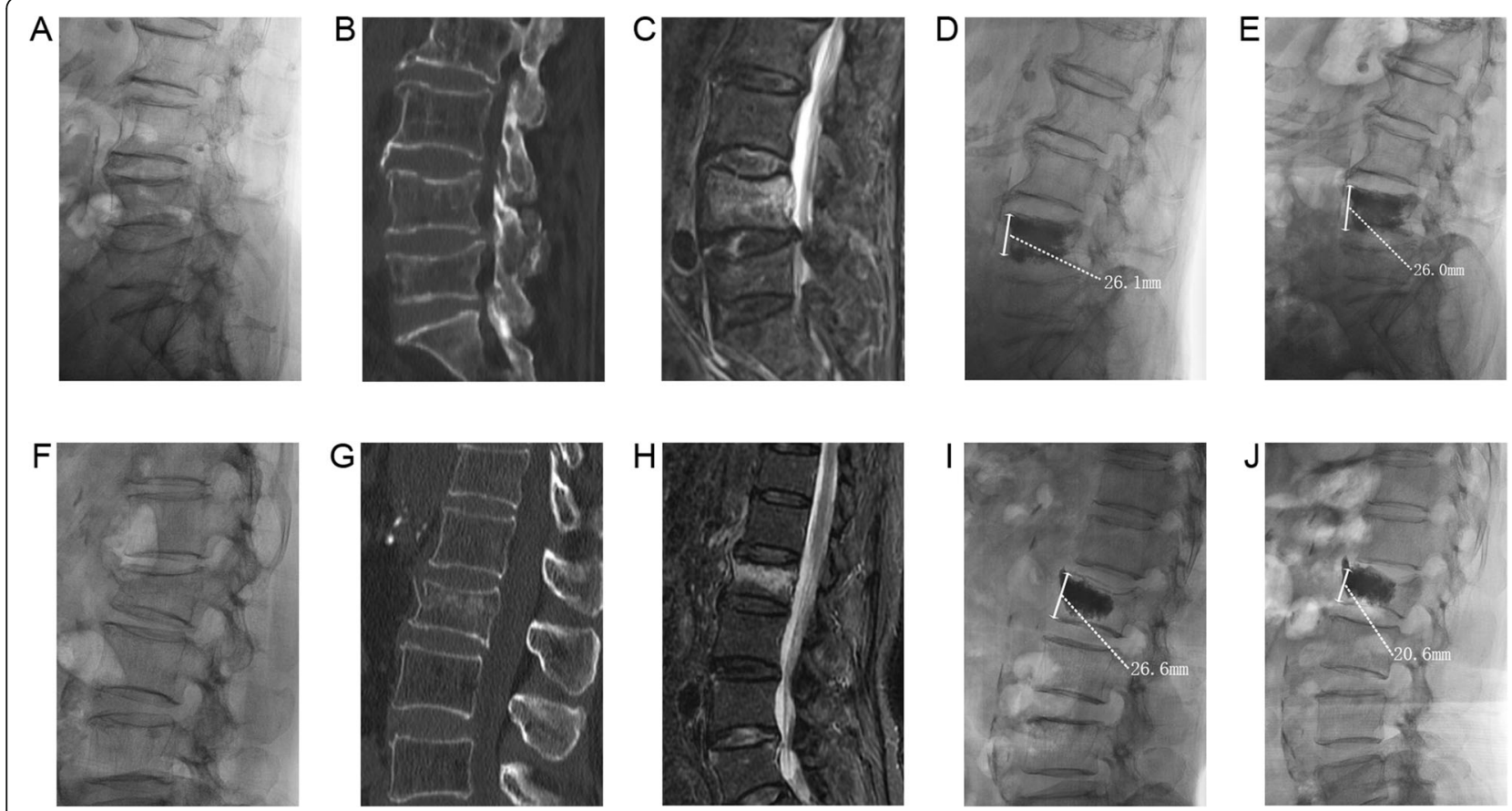

Fig. 2 Typical cases. A 71 years old female patient, preoperative $x$-ray (a), CT (b), MRI (c) showed acute OVCF of L4. X-ray (d) at $24 \mathrm{~h}$ post operation showed that the cement was in close contact with the upper and lower endplates, and X-ray (e) at 12 months post operation showed that the vertebral height was maintained well. A 62 years old female patient, preoperative $x$-ray (f), CT (g), MRI (h) showed acute OVCF of L2. The X-ray (i) at $24 \mathrm{~h}$ post operation showed that the cement did not contact the lower endplate. The X-ray (j) at 6 months post operation showed that the height of the vertebral body was lost and the vertebral body was recompressed

Analysis in subgroup B revealed the recompression rate was higher in group B3 when bone cement contacted none of the endplate, but there is no statistical difference, which may due to the relatively small sample size. The upper and lower endplates are equally important. The lack of contact between the bone cement and any endplate will result in an unfilled vulnerable area, where recompression usually happened.

In our study, regardless of the type of cement distribution, it has obvious benefits for short term pain relief and recovery of vertebral height. And this is consistent with previous clinical experience [20]. However, in the long-term follow up, the degree of pain in group A were significantly lower than group B. Ye revealed insufficient filling of bone cement was associated with chronic lower back pain [21]. Recompression may lead to changes in spinal balance, local kyphosis, and consequently chronic pain. Especially when there is a fracture in vertebral endplate, if the cement is not well connected with the endplate, it will provide insufficient support and lead to the continuous compression of the fracture vertebral body, which is the reason for the persistence of postoperative pain [22]. He found that the long-term effect of H-type distribution of bone cement is better than O-type distribution, which is related to the closer contact between bone cement, endplates and cancellous bone in $\mathrm{H}$-type distribution [23]. Our study and previous literatures show that if the bone cement is evenly distributed and closely contacted with the upper and lower endplates, it can better maintain the strength and height of the vertebral body, reduce recompression risk and eventually improve the patients' chronic back pain.

It is not an ideal way to obtain a wide distribution of bone cement by increasing excessive cement volume. Because laboratory based biomechanical study found that the stiffness of the injured vertebral body can be restored when the volume of bone cement reaches approximately $15 \%$ of the vertebral body. If the volume of bone cement injected is increased beyond this value, there is no significant benefit, and it may cause asymmetric distribution of bone cement and excessive rigidity of the vertebral body [24]. The clinical usage of cement volume can be excessive than $15 \%$, nonetheless, few significant benefits have been shown when the volume reaches to beyond $24 \%$ of the vertebral body, at which point that can already effectively relieve the pain $[25$, 26]. In our study, no significantly difference of bone cement volume was found between groups. Additionally, an increase in the cement volume may increase the risk of cement leakage [27-29]. Bone cement volume is only weakly related to the effect of the operation [30] and it is not advisable to increase the volume of bone cement 
excessively. Compared with percutaneous kyphoplasty (PKP), PVP may achieve better cement distribution. Loss of vertebral height was more likely after PKP than PVP $[31,32]$. Because balloons squeeze cancellous bone around during expansion in PKP, creating a "cavity". Cement tends to distribute in this low-pressure cavity without infiltrating into the surrounding bone, making it difficult for cancellous bone to bond tightly, and this mass-like cement distribution has also been proved to be risk factor of recompression [33].

In addition, when puncturing bilaterally, the angle of puncture needle can be adjusted so that the two puncture needles point to the upper or lower endplate respectively [34], using high-viscosity cement [35], using hydraulic assistant device to inject bone cement slowly and uniformly may be more beneficial for a better distribution of bone cement [36]. High-viscosity cement has the advantages of fast bonding with bone, long working time window and low polymerization temperature [37]. A meta-analysis found that high-viscosity cement has significant advantages in pain improvement, recovery of cobb angle and cement leakage comparing with lowviscosity cement [38].

There are some limitations in this study, the retrospective study and relatively small sample size may produce some bias. Prospective studies with large numbers of cases are needed to further clarify the relationship between cement distribution and surgical outcome.

\section{Conclusions}

Whether or not the cement is in full contact with the upper and lower endplates, it can have a good immediate analgesic effect. However, if the bone cement fully contacted both the upper and lower endplates, it can better restore the strength of the vertebral body, and then better maintain the height of the vertebral body, reduce the risk of the vertebral body recompression, and its long-term effect is better.

\section{Abbreviations}

PVP: Percutaneous vertebroplasty; OVCF: Osteoporotic vertebral compression fracture; VAS: Visual analogue scale; AVHRR: Anterior vertebral height restoration ratio; AVHLR: Anterior vertebral height loss ratio; BMI: Body mass index; BMD: Bone mineral density; PKP: Percutaneous kyphoplasty

\section{Acknowledgements}

Not applicable.

\section{Authors' contributions}

$T L$ collected, analyzed, and interpreted the data and wrote the draft. WBT, GZQ, CZQ performed the surgery, designed the protocol, revised the draft. All the authors have read and approved the final manuscript.

\section{Funding}

Supported by National Key R\&D Program of China (2018YFB1307600).

\section{Availability of data and materials}

The datasets used during the current study are available from the corresponding author on reasonable request.

\section{Ethics approval and consent to participate}

The experimental protocol was established, according to the ethical guidelines of the Helsinki Declaration and was approved by the Human Ethics Committee of Peking University International Hospital. Written informed consent was obtained from each participant.

\section{Consent for publication}

Written informed consent for publication was obtained from each participant.

\section{Competing interests}

Tan Lei, Wen Bingtao, Guo Zhaoqing, Chen Zhongqiang declare that they have no conflict of interest.

\section{Author details}

'Department of Orthopaedics, Peking University International Hospital, Life Park Road No.1 Life Science Park of Zhong Guancun, Changping District, Beijing 102206, China. ${ }^{2}$ Department of Orthopaedics, Peking University Third Hospital, 49 North Garden Road, Haidian District, Beijing 100191, China.

Received: 7 May 2020 Accepted: 4 August 2020

Published online: 13 August 2020

\section{References}

1. Chen D, An ZQ, Song S, Tang JF, Qin H. Percutaneous vertebroplasty compared with conservative treatment in patients with chronic painful osteoporotic spinal fractures. J Clin Neurosci. 2014;21:473-7.

2. Liu J, Li X, Tang D, Cui X, Li X, Yao M, et al. Comparing pain reduction following vertebroplasty and conservative treatment for osteoporotic vertebral compression fractures: a meta-analysis of randomized controlled trials. Pain Physician. 2013;16:455-64.

3. Itshayek E, Miller P, Barzilay Y, Hasharoni A, Kaplan L, Fraifeld S, et al. Vertebral augmentation in the treatment of vertebral compression fractures: review and new insights from recent studies. J Clin Neurosci. 2012;19:786-91.

4. Karmakar A, Acharya S, Biswas D, Sau A. Evaluation of percutaneous Vertebroplasty for Management of Symptomatic Osteoporotic Compression Fracture. J Clin Diagn Res. 2017;11:RC07-10.

5. Xie L, Zhao ZG, Zhang SJ, Hu YB. Percutaneous vertebroplasty versus conservative treatment for osteoporotic vertebral compression fractures: An updated meta-analysis of prospective randomized controlled trials. Int J Surg. 2017:47:25-32.

6. Yang JS, Liu JJ, Chu L, Li J, Chen C, Chen H, et al. Causes of residual Back pain at early stage after percutaneous Vertebroplasty: a retrospective analysis of 1,316 cases. Pain Physician. 2019;22:E495-503.

7. Molloy S, Mathis JM, Belkoff SM. The effect of vertebral body percentage fill on mechanical behavior during percutaneous vertebroplasty. Spine. 2003;28: 1549-54.

8. Lin J, Qian L, Jiang C, Chen X, Feng F, Lao L. Bone cement distribution is a potential predictor to the reconstructive effects of unilateral percutaneous kyphoplasty in OVCFs: a retrospective study. J Orthop Surg Res. 2018;13:140.

9. Yang S, Chen C, Wang H, Wu Z, Liu L. A systematic review of unilateral versus bilateral percutaneous vertebroplasty/percutaneous kyphoplasty for osteoporotic vertebral compression fractures. Acta Orthop Traumatol Turc. 2017:51:290-7.

10. Chen YC, Zhang L, Li EN, Ding LX, Zhang GA, Hou Y, et al. Unilateral versus bilateral percutaneous vertebroplasty for osteoporotic vertebral compression fractures in elderly patients: A meta-analysis. Medicine (Baltimore). 2019;98:e14317.

11. Yu WB, Jiang $X B$, Liang $D, X u W X, Y e L Q$, Wang J. Risk factors and score for recollapse of the augmented vertebrae after percutaneous vertebroplasty in osteoporotic vertebral compression fractures. Osteoporos Int. 2019;30:423-30.

12. Heo DH, Chin DK, Yoon YS, Kuh SU. Recollapse of previous vertebral compression fracture after percutaneous vertebroplasty. Osteoporos Int. 2009;20:473-80.

13. Lavelle WF, Cheney R. Recurrent fracture after vertebral kyphoplasty. Spine J. 2006;6:488-93.

14. Yu W, Liang D, Yao Z, Qiu T, Ye L, Huang X, et al. Risk factors for recollapse of the augmented vertebrae after percutaneous vertebroplasty for 
osteoporotic vertebral fractures with intravertebral vacuum cleft. Medicine (Baltimore). 2017:96:e5675.

15. Gaughen JR Jr, Jensen ME, Schweickert PA, Marx WF, Kallmes DF. The therapeutic benefit of repeat percutaneous vertebroplasty at previously treated vertebral levels. AJNR Am J Neuroradiol. 2002;23:1657-61.

16. Xu K, Li YL, Song F, Liu HW, Yang HD, Xiao SH. Influence of the distribution of bone cement along the fracture line on the curative effect of vertebral augmentation. J Int Med Res. 2019;47:4505-13.

17. Chevalier Y, Pahr D, Charlebois M, Heini P, Schneider E, Zysset P. Cement distribution, volume, and compliance in vertebroplasty: some answers from an anatomy-based nonlinear finite element study. Spine. 2008;33:1722-30.

18. Kim YY, Rhyu KW. Recompression of vertebral body after balloon kyphoplasty for osteoporotic vertebral compression fracture. Eur Spine J. 2010;19:1907-12.

19. Liang D, Ye LQ, Jiang XB, Yang P, Zhou GQ, Yao ZS, et al. Biomechanical effects of cement distribution in the fractured area on osteoporotic vertebral compression fractures: a three-dimensional finite element analysis. J Surg Res. 2015;195:246-56.

20. Watts NB, Harris ST, Genant HK. Treatment of painful osteoporotic vertebral fractures with percutaneous vertebroplasty or kyphoplasty. Osteoporos Int. 2001;12:429-37.

21. Ye LQ, Liang JXB, Yao ZS, Lu H, Qiu T, et al. Risk factors for the occurrence of insufficient cement distribution in the fractured area after percutaneous vertebroplasty in osteoporotic vertebral compression fractures. Pain Physician. 2018;21:E33-42.

22. Jacobson RE, Palea O, Granville M. Progression of vertebral compression fractures after previous vertebral augmentation: technical reasons for recurrent fractures in a previously treated vertebra. Cureus. 2017;9:e1776.

23. He S, Zhang $Y, L v N$, Wang $S$, Wang $Y$, Wu S, et al. The effect of bone cement distribution on clinical efficacy after percutaneous kyphoplasty for osteoporotic vertebral compression fractures. Medicine (Baltimore). 2019;98:e18217.

24. Liebschner MA, Rosenberg WS, Keaveny TM. Effects of bone cement volume and distribution on vertebral stiffness after vertebroplasty. Spine. 2001;26: 1547-54.

25. Belkoff SM, Mathis JM, Jasper LE. Deramond. The biomechanics of vertebroplasty. The effect of cement volume on mechanical behavior. Spine. 2001;26:1537-41.

26. Nieuwenhuiise MJ, Bollen L, van Erkel AR, Dijkstra PD. Optimal intravertebral cement volume in percutaneous vertebroplasty for painful osteoporotic vertebral compression fractures. Spine. 2012;37:1747-55.

27. Lin $D$, Hao J, Li L, Wang L, Zhang H, Zou W, et al. Effect of bone cement volume fraction on adjacent vertebral fractures after unilateral percutaneous kyphoplasty. Clin Spine Surg. 2017;30:E270-5.

28. Jia P, Tang H, Chen H, Bao L, Feng F, Yang H, et al. Prophylactic vertebroplasty procedure applied with a resorbable bone cement can decrease the fracture risk of sandwich vertebrae: long-term evaluation of clinical outcomes. Regen Biomater. 2017:4:47-53.

29. Zhang H, Xuan J, Chen TH, Chen ZX, Sun LJ, Tian NF, et al. Projection of the Most anterior line of the Spinal Canal on lateral radiograph: An anatomic study for percutaneous Kyphoplasty and percutaneous Vertebroplasty. J Investig Surg. 2020;33:134-40.

30. Fu Z, Hu X, Wu Y, Zhou Z. Is there a dose-response relationship of cement volume with cement leakage and pain relief after vertebroplasty? Dose Response. 2016;14:1559325816682867.

31. Kim MJ, Lindsey DP, Hannibal M, Alamin TF. Vertebroplasty versus kyphoplasty: biomechanical behavior under repetitive loading conditions. Spine. 2006;31:2079-84.

32. Wilke HJ, Mehnert U, Claes LE, Bierschneider MM, Jaksche H, Boszczyk BM. Biomechanical evaluation of vertebroplasty and kyphoplasty with polymethyl methacrylate or calcium phosphate cement under cyclic loading. Spine. 2006;31:2934-41.

33. He D, Lou C, Yu W, Zhu K, Wu Z, Liu F, et al. Cement distribution patterns are associated with recompression in cemented vertebrae after percutaneous Vertebroplasty: a retrospective study. World Neurosurg. 2018; 120:1-7.

34. Nieuwenhuijse MJ, van Erkel AR, Dijkstra PD. Percutaneous vertebroplasty in very severe osteoporotic vertebral compression fractures: feasible and beneficial. J Vasc Interv Radiol. 2011;22:1017-23.

35. Baroud G, Crookshank M, Bohner M. High-viscosity cement significantly enhances uniformity of cement filling in vertebroplasty: An experimental model and study on cement leakage. Spine. 2006;31:2562-8.
36. Jeong YH, Lee CJ, Yeon JT, Bae J, Choi E, Lee PB, et al. Insufficient penetration of bone cement into the trabecular bone_ a potential risk for delayed bone cement displacement after kyphoplasty? Reg Anesth Pain Med. 2016;41:616-8.

37. Zhang L, Wang J, Feng $X$, Tao Y, Yang J, Wang Y, et al. A comparison of high viscosity bone cement and low viscosity bone cement vertebroplasty for severe osteoporotic vertebral compression fractures. Clin Neurol Neurosurg. 2015;129:10-6.

38. Zhang ZF, Huang $\mathrm{H}$, Chen $\mathrm{S}$, Liu DH, Feng $\mathrm{YH}$, Xie $\mathrm{CL}$, et al. Comparison of high- and low-viscosity cement in the treatment of vertebral compression fractures: A systematic review and meta-analysis. Medicine (Baltimore). 2018; 97:e0184.

\section{Publisher's Note}

Springer Nature remains neutral with regard to jurisdictional claims in published maps and institutional affiliations.
Ready to submit your research? Choose BMC and benefit from:

- fast, convenient online submission

- thorough peer review by experienced researchers in your field

- rapid publication on acceptance

- support for research data, including large and complex data types

- gold Open Access which fosters wider collaboration and increased citations

- maximum visibility for your research: over $100 \mathrm{M}$ website views per year

At BMC, research is always in progress.

Learn more biomedcentral.com/submissions 